\title{
Mucormicose rinocerebral associada a trombose de seio cavernoso: Relato de Caso
}

\author{
Rhinocerebral Mucormycosis Associated with Cavernous Sinus Thrombosis: Relato de Caso
}

Nina Feital Montezzi ${ }^{\dagger}$, Ana Beatriz Calmon Nogueira da Gama Pereira ${ }^{\ddagger}$, Karen Batista Vogas ${ }^{\S}$, Carolina Ferraz S. Veigall, Bruno Barbosa Cardoso dos Santos ${ }^{\dagger}$, Mariana Marques Rechuan ${ }^{\dagger}$

Como citar esse artigo. Montezzi,

N.F.; Pereira, A.B.C.N.G.; Vogas, K..B.; Veiga, C.F.S; dos Santos, B.B.C.; Rechuan, M.M. Mucormicose rinocerebral associada a trombose de seio cavernoso: Relato de Caso. Revista de Saúde. 2020 Jan./Jun.; 11 (1): 10-14

\begin{abstract}
Resumo
Mucormicose rinocerebral é uma infecção fúngica rara e invasiva da cavidade nasal e seios da face, podendo evoluir para áreas de necroses, fístulas, acometimento orbital e cerebral. Acomete principalmente indivíduos imunodeprimidos eportadores de cetoacidose diabética, com letalidade de aproximadamente $50 \%$ dos casos. É considerada uma emergência médica. Uma possível complicação intracraniana é a trombose do seio cavernoso, com prognóstico limitado. É relatado um paciente imunocompetente, 62 anos, diabético,com mucormicose, quadro de rinossinusite fúngica, além da trombose de seio cavernoso com paralisia facial e dos nervos oculomotor, troclear e abducente. Foi tratado com Anfotericina B, Vancomicina, Cefepime, Enoxaparina, com posterior desbridamento cirúrgico e evoluiu com reversão dos sintomas neurológicos. Esse caso requer diagnóstico complexo e tratamento imediato devido a sua alta letalidade (50\%). É essencial considerar essa hipótese diagnóstica em pacientes diabéticos e que apresentam mau controle glicêmico ou em cetoacidose diabética com sinusite crônica ou não responsiva ao tratamento padrão.
\end{abstract}

Palavras-chave: Mucormicose, Sinusite, Paralisia Facial.

\begin{abstract}
Rhinocerebralmucormycosis is a rare and invasive fungal infection of the nasal cavity and sinuses, and may progress to areas of necrosis, fistulae, orbital and cerebral involvement. It mainly affects immunosuppressed individuals and diabetic ketoacidosis, with a lethality of approximately $50 \%$ of cases. It is considered a medical emergency. A possible intracranial complication is cavernous sinus thrombosis, with a limited prognosis. We report a 62-year-old diabetic immunocompetent patient with mucormycosis, fungal rhinosinusitis, and cavernous sinus thrombosis with facial hemiparesis, and oculomotor, trochlear, and abducens nerves paralysis. He was treated with amphotericin B, vancomycin, cefepime, enoxaparin, with subsequent surgical debridement and evolved with reversal of neurological symptoms. This case requires complex diagnosis and immediate treatment due to its high lethality $(50 \%)$. It is essential to consider this diagnostic hypothesis in diabetic patients with poor glycemic control or diabetic ketoacidosis with chronic sinusitis or unresponsive to standard treatment.

Keywords: Mucormycosis, Sinusitis, Facial Paralysis.
\end{abstract}

\section{Introdução}

Mucormicosese referea infecções fúngicas causadas por fungo da classe Zygomycetes eordem Mucorales, tendo como representante mais frequente o Rhizopusoryzae, responsável por aproximadamente $60 \%$ das formas de mucormicose. Este fungo pode ser encontradoem pães, frutas, no solo e em matéria orgânica em decomposição ${ }^{1}$. Essa doença pode apresentar uma forma pulmonar, mais prevalente em pacientes neutropênicos e com câncer hematológico ${ }^{2}$, além das formas gastrointestinal, neurológica, cutânea ou sistêmica ${ }^{3}$. A mais comum é a rinocerebral,que está mais associada a cetoacidose diabética. O fungo invade a mucosa nasal,seguido dos seios da face, acompanhado de erosão em palato duro, órbita, chegando ao encéfalo ${ }^{4}$ através da lâmina cribiforme ou por trombose arterial do $\mathrm{SNC}^{5}$.

Sabe-se que qualquer imunodeficiência pode precipitar o quadro. Entre elas estão pacientes transplantados com leucemias, linfomas, queimaduras extensas, diabetes mellitus, insuficiência renal ou hepática, em quimioterapia ou em uso de altas doses de corticoides ou imunossupressores. ${ }^{5}$ Entretanto, a mucormicose não é comum nos pacientes HIV positivos, já que a imunidade mediada por células $T$ não é considerada um fator importante na patogenia ${ }^{1}$.

É uma infecção bastante rara $^{2}$. Segundo Yohia et al., ${ }^{6}$ os principais sintomas encontrados foram: febre $(44 \%)$, necrose ou úlcera nasal $(38 \%)$, edema facial ou periorbital (34\%), redução da acuidade visual $(30 \%)$, oftalmoplegia $(29 \%)$, sinusite $(26 \%)$ e cefaleia

\footnotetext{
Afiliação dos autores: $\uparrow$ Acadêmica do curso de Medicina da Universidade de Vassouras; Vassouras, RJ, Brasil

\$ Médica Neurologista do Hospital Universitário de Vassouras; Vassouras, RJ, Brasil

§ Médica coordenadora do serviço de Clínica Médica do Hospital Universitário de Vassouras; Vassouras, RJ, Brasil

\| Médica Residente de Clínica Médica do Hospital Universitário de Vassouras; Vassouras, RJ, Brasil

* Email de correspondência: ninafmontezzi@gmail.com
} 
(25\%). Podem aparecer também súbita dor facial ou orbital unilateral, obstrução e secreção nasal necrótica, evoluindo com lesão lítica na mucosa, celulite orbitária, amaurose e anestesia de córnea.

São achados comuns à tomografia computadorizada a invasão tecidual, necrose de seios da face acompanhado de erosões ósseas e trombose do seio cavernoso. Entretanto, a Ressonância Nuclear Magnética é considerada o melhor método de avaliação das alterações precoces nos vasos, como a trombose da artéria carótida e do seio cavernoso, além de estudar possível extensão intracraniana da doença ${ }^{2}$.

O diagnóstico baseia-se na visualização de hifas no exame direto e histopatológico das lesões e biópsias do material necrótico ${ }^{1}$. Para tal, deve ser solicitado especificamente essa pesquisa.

O tratamento se baseia no diagnóstico precoce, desbridamento cirúrgico agressivo e terapia com anfotericina B sistêmica ${ }^{1,3}$ em doses elevadas $(0,8$ a $1,5 \mathrm{mg} / \mathrm{kg} / \mathrm{dia}$ ), sendo potencialmente nefrotóxica. Entretanto, melhora a sobrevida dos pacientes em tratamento de $37 \%$ para $79 \%$ em diabéticos e de $0 \%$ para $47 \%$ nos não-diabéticos ${ }^{1}$.

A doença possui prognóstico reservado, tendo a zicomicose rinocerebral taxa de mortalidade de, aproximadamente, $50 \%{ }^{6}$. Esta evolução potencialmente fatal se deve ao tropismo vascular desses fungos, que invadem as artérias, causando trombose e lesões isquêmicas ${ }^{4}$. Sinais de mau prognóstico incluem necrose facial, deformidade nasal e hemiplegia ${ }^{3}$.

A principal complicação intracraniana da mucormicose é a Trombose do Seio Cavernoso. As queixas iniciais são de cefaleia, dor retro-orbital, edema periorbitário, proptose, diplopia e redução da acuidade visual. $\mathrm{O}$ agente causador mais comum é o Staphylococcus aureus, mas pode ser causado por outras bactérias ou fungos ${ }^{4}$.

$\mathrm{O}$ presente relato de caso visa divulgar para a sociedade científica um caso o de uma doença rara e invasiva que exige diagnóstico e tratamento clínico e cirúrgico rápidos, sabendo que pode ser subdiagnosticada em grande parte das vezes, devido a falta de conhecimento.

\section{Material e Métodos}

Foi feito relatado o caso de um paciente internado no setor de Clínica Médica do Hospital Universitário de Vassouras durante 02/03/19 a 03/05/19. Dados foram obtidos através do prontuário, após deferida a Solicitação de Campo, e foi assinado o Termo de Consentimento Livre e Esclarecido pelo paciente. O relato de caso pertence ao Projeto de Pesquisa: "Rastreamento de Doenças Neurológicas no Município de Vassouras", aprovado pelo Comitê de Ética em Pesquisa.

\section{Relato de caso}

Paciente, 62 anos, homem, branco, com antecedentes de diabetes mellitus tipo 1 em uso de 70 UI de insulina NHP (40 UI pela manhã e 30 UI à noite), metformina $850 \mathrm{mg}$ duas vezes ao dia, e glibenclamida $8 \mathrm{mg} /$ dia, além de hipertensão arterial sistêmica sem tratamento há 4 anos, fibrilação atrial crônica e doença pulmonar obstrutiva crônica não tratadas. Relata difícil controle glicêmico, culminando com a amputação de hálux esquerdo, quarto e quinto pododáctilos à direita previamente. Apresentou um quadro de rinosinusite e otite, acompanhadas de dor em fisgadas em hemiface esquerda e cefaleia há 3 semanas, assim como escotomas cintilantes na visão esquerdae diplopia após a remoção de um corpo estranho em ouvido esquerdo. Em uso de Levofloxacino.PA=140X90 $\mathrm{mm} \mathrm{Hg}, \mathrm{FC}=77 \mathrm{bpm}$, Tax= $36,5^{\circ} \mathrm{C}, \mathrm{FR}=19 \mathrm{irpm}, \mathrm{HGT}=244 \mathrm{mg} / \mathrm{dL}$. À otoscopia saída de secreção purulenta à esquerda. Apresentava anisocoria e alteração na mobilidade ocular (paralisia do abducente). Os exames laboratoriais da internação mostraram hemograma com discreta anemia, 7.310 leucócitos com $8 \%$ bastões, $4 \%$ eosinófilos, 14\% linfócitos. Bioquímica: $\mathrm{Na} 128 \mathrm{mmol} / \mathrm{L}, \quad \mathrm{K} \quad 3,9$ $\mathrm{mmol} / \mathrm{L}$, Ureia $17 \mathrm{mg} / \mathrm{dL}$, Creatinina $0,7 \mathrm{mg} / \mathrm{dL}$. PCR 138,2. Função hepática normal. Duas hemoculturas e urinocultura negativas. No EAS havia glicose $(3+/ 4+)$ e corpos cetônicos $(2+/ 4+)$. Evoluiu com hemiparesia facial esquerda dolorosa,oftalmoplegia completa com anisocoria, amaurose,ptose e proptose palpebrais ipsilateraisàlesãonomesmodia.Aoexameoftalmológico: fundo de olho normal. Neurologicamente: paralisia do terceiro, quarto e sexto nervos cranianos. Visualizou-se uma lesão ulcerativa em palato com comunicação para a cavidade nasal, em que foi feita a biópsia com análise histopatológica, com posterior resultado contendo células inflamatórias, sugestivo de úlcera crônica, ausência de malignidades. Não foi visualizado hifas ou elementos fúngicos na coloração padrão. A tomografia computadorizada (figura 1) mostrou velamento em seio maxilar, das células etmoidais à esquerda, erosões ósseas em parede dos seios, além de espessamento e realce do contraste na parede lateral do seio cavernoso esquerdo (figura 2).

Foi iniciado tratamento empírico para rinosinusite bacteriana grave com ceftriaxone por 11 dias, sem melhora clínica. Dosou-se anti-HIV, p- ANCA,cANCA e FAN não reagentes, afastando a hipótese de Granulomatose de Wegener.

No décimo sexto dia de internação, apresentou leucopenia (2050 leucócitos $/ \mathrm{mm}^{3}$ ) e a hemocultura positiva para $S$. aureus MRSA. Foi pensada a hipótese diagnóstica de mucormicose associada a trombose de seio cavernoso, iniciando Anfotericina B $1 \mathrm{mg} / \mathrm{kg} / \mathrm{dia}$, ou $75 \mathrm{mg}$ em Bomba Infusora $120 \mathrm{~mL} / \mathrm{h}$, Cefepime $2 \mathrm{~g}$ 
diluído IV 8/8h, Vancomicina $1 \mathrm{~g}$ de 12/12h e Clexane $60 \mathrm{mg} \mathrm{SC}$ de 12/12h. O uso de Cefepime se justifica por ampliar a cobertura bacteriana diante da gravidade do quadro. Além das medicações para controle da pressão arterial (losartana 50mg 12/12h e metoprolol 50mg 1 vez ao dia) e glicemia (insulina regular conforme HGT). Paciente relatou melhora dos sintomas da rinossinusite, alívio da sensação de dor e peso em face, sem otorreia. No décimo dia de Anfotericina B, evoluiu com injúria renal aguda, creatinina $2,7 \mathrm{mg} / \mathrm{dL}$, ureia $75 \mathrm{mg} / \mathrm{dL}$ continuamente se elevando até valores de creatinina 5,5 $\mathrm{mg} / \mathrm{dL}$ e ureia $109 \mathrm{mg} / \mathrm{dL}$. Necessitou de hemodiálise três vezes por semana, por 4 semanas e reajuste das doses das medicações. Os sintomas oculares e neurológicos foram regredindo até completa remissão, assim como a recuperação da função renal. O tratamento foi concluído com 28 dias de medicação e posterior drenagem cirúrgica de seio da face com sinusoidectomia esquerda,sem intercorrências. Após recuperação cirúrgica, o paciente foi liberado para acompanhamento ambulatorial com a Clínica Médica e a Nefrologia, retornando a sua vida e atividades habituais.

\section{Discussão}

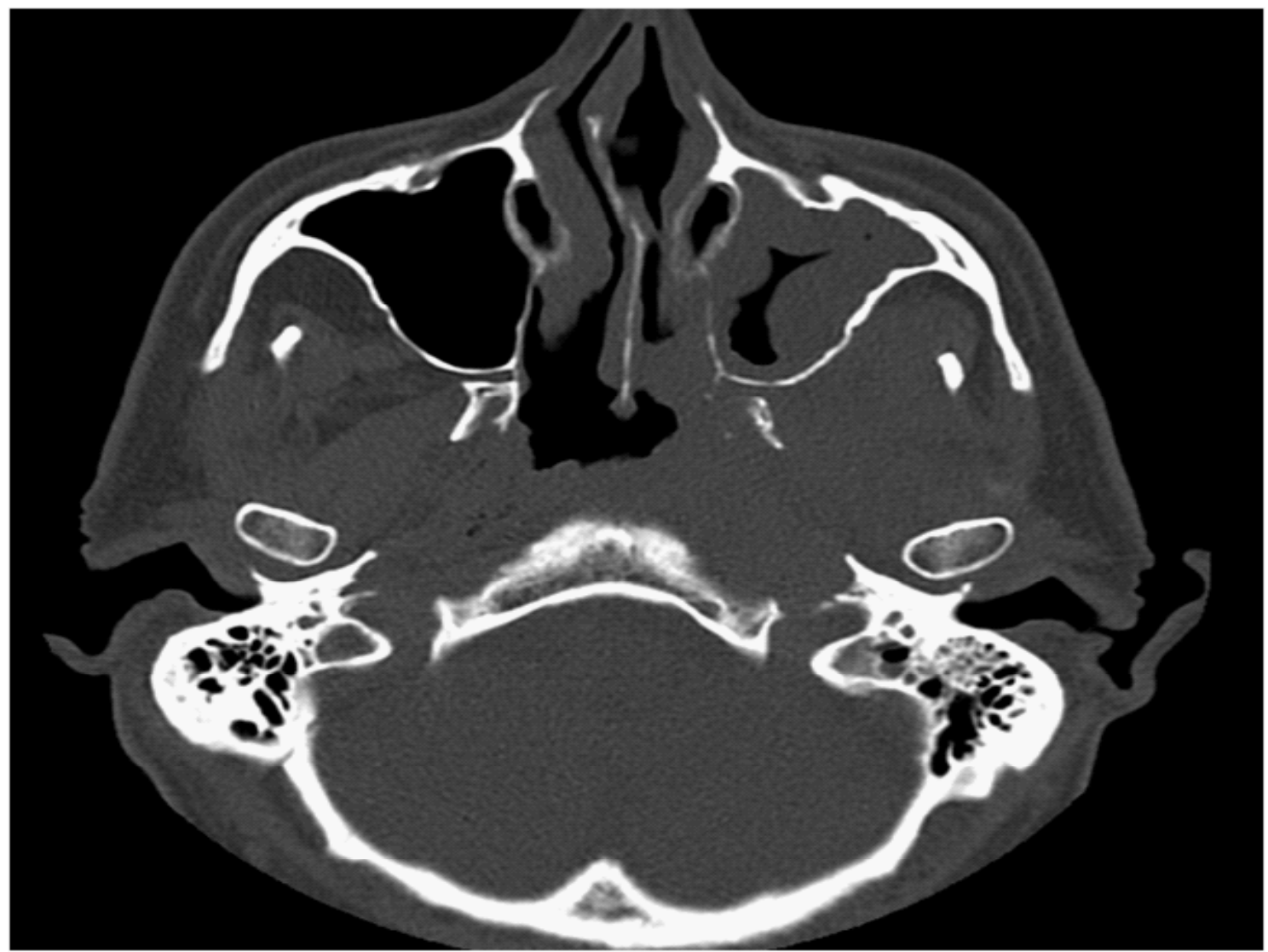

Figura 1: Tomografia computadorizada evidenciando sinusite de seio maxilar esquerdo.

No caso, o paciente é diabético de difícil controle com glicemias extremamente elevadas na internação e na história colhida, mas não apresentava instabilidade clínica, sinais de acidose ou imunodepressão, o que não é comum de ser encontrado na bibliografia. O resultado da biópsia de palato foi de células inflamatórias, o que não exclui a possibilidade de infecção fúngica. Como não era o diagnóstico principal inicial, não foi pedida coloração específica para fungos, como a técnica Grocott1 nem a imunofluorescência indireta, que faria o diagnóstico etiológico ${ }^{1}$. Mesmo assim, o diagnóstico pode ser fechado pela clínica típica, exames de imagem e resposta clínica favorável ao tratamento.

$\mathrm{O}$ diagnóstico precoce e a cirurgia são fundamentais para uma boa evolução da mucormicose $\mathrm{e}^{1,2}$.

No caso tratado, a demora na realização da cirurgia e no resultado anatomopatológico da biópsia de palato fez com que se priorizasse o tratamento empírico, que obteve resultado de cura para o paciente. Foi usada Anfotericia B $1 \mathrm{mg} / \mathrm{kg} / \mathrm{dia}$, uma droga nefrotóxica, que causou injúria renal aguda, necessitando de hemodiálise. Uma alternativa seria a anfotericina B lipossomal, que permite atingir maiores doses da medicação com menos agressão renal, todavia é uma medicação cara e de difícil 


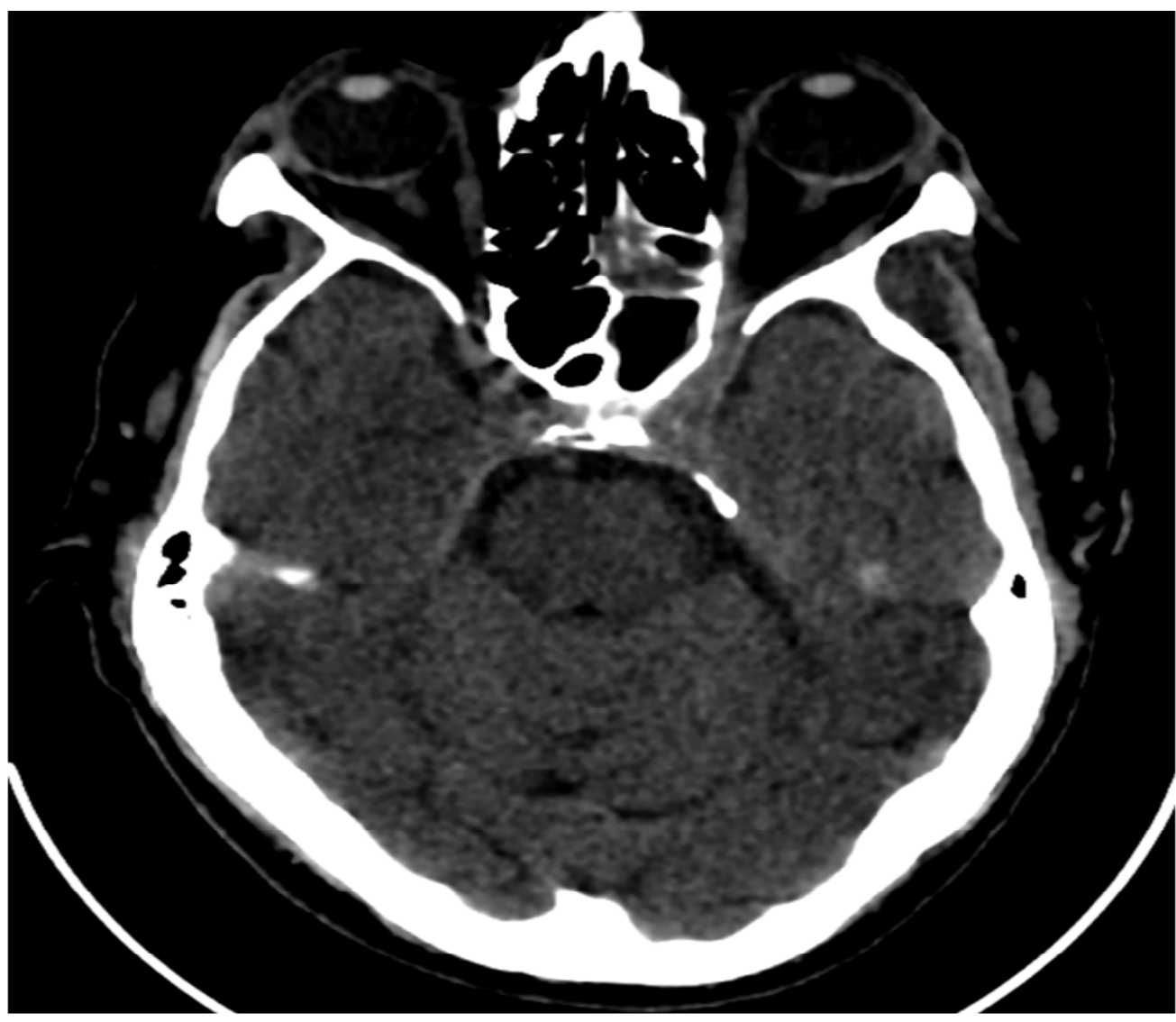

Figura 2: Tomografia computadorizada evidenciando espessamento e realce do contraste na parede lateral do seio cavernoso esquerdo.

acesso ${ }^{7,8}$.Essa droga apresentou melhores resultados do que a Anfotericina B não-lipossomal no estudo de Leenders et $a l^{8}$.

Outras opções para tratamento que se encontram na bibliografia sãoa câmara hiperbárica de oxigênio, que ajuda na recuperação e evita o crescimento de lesões necróticas ${ }^{9}$ e cirurgia endoscópica ${ }^{3}$, que se mostraram promissoras. Énecessário saberidentificar precocemente o quadro e intervir na sua história natural.

As queixas iniciais de Trombose do Seio Cavernoso dor retro-orbital (edema periorbital, quemose, proptose, ptose palpebral e diplopia) são sintomas inespecíficos e podem estar presentes em outras afecções, como na celulite orbitária. Entretanto, se o paciente apresenta sepse, paralisia de pares cranianos ou envolvimento ocular bilateral é um sinal cardinal para o diagnóstico da TSC4. No paciente em estudo, foi feita uma hemocultura positiva para Staphylococcus aureus, agente patogênico mais comum encontrado nesses casos $^{3}$. Por esse motivo foi associada ao tratamento a Vancomicina e a Enoxaparina, que conseguiram reverter essa trombose. A ressonância magnética feita no final do tratamento mostra o seio cavernoso sem trombo, o que coincidiu com a melhora dos sintomas neurológicos.

\section{Conclusão}

A mucormicose é uma infecção fúngica rara, invasiva, potencialmente fatal. Por isso é importante salientar que todo doente imunodeprimido, em especial os com cetoacidose diabética e rinossinusite sem melhora do quadro com tratamento padrão, deve-se considerar o diagnóstico de mucormicose. Na suspeita, a terapêutica disponível deve ser prontamente iniciada, o que melhora a taxa de sobrevida.

\section{Referências}

1. Severo CB, Guazzelli LS, Severo LC. Capítulo 7 - Zigomicose. J. bras. pneumol. 2010 Feb; 36(1): 134-141.

2. Xavier SD, Korn GP, Granato L. Mucormicoserinocerebral: apresentação de caso com sobrevida e revisão de literatura. Rev. Bras. Otorrinolaringol. 2004 Oct; 70(): 710-714.

3. Santana Nícia O. R., Pinheiro Gustavo B., KehrleHelga M., Faria Isnaldo P. de, Estrella Cláudio N. Mucormicose de seios paranasais e órbitas em paciente imunocompetente: relato de caso e revisão de literatura. Rev. Bras. Otorrinolaringol. $2001 \mathrm{Sep} ; 67(5)$ : 727-730.

4. HaberDM, FernandesAM, Neto DDS, SchiavettoRR. Mucormicose RinoÓrbito-Cerebral Associada com Trombose do Seio Cavernoso: Relato de Caso. Arq. Int. Otorrinolaringol. 2008 Mai; 12 (4): 574-578

5.Vilela VM, Marques HC, Carvalho REDS, Zavelinske E, Duque AGS, Dutra BL. Síndrome do seio cavernoso secundária a mucormicoserinoorbitocerebral. Radiol Bras. 2013 June; 46(3): 187-189.

6. Yohia RA, Bullock JD, AzizAA, Market RJ. Survival factors in rhinoorbital cerebral mucormycosis. SurvOphthalmol.1994 Jul-Aug;39(1):3-22. 
7. Strasser MD, Kennedy RJ, Adam RD. Rhinocerebralmucormycosis therapy with amphotericin B lipid complex. Arch Intern Med 1961 Feb;156(3):337339

8. Leenders AC, Daenen S, Jansen RL et al. Liposomal amphotericin B compared with amphotericin B deoxycholate in the treatment of documented and suspected neutropenia- associated invasive fungal infections. Br J Haematol. 1998 Oct;103(1):205-12.

9. Couch L, Theilen F, Mader JT. Rhinocerebralmucormycosis with cerebral extension successfully treated with adjuntive hyperbaric oxygen. Arch Otolaryngol. $1988 \mathrm{Jul} ; 114(7): 791-4$ 